\title{
Spatial Optical Solitons in Bulk Nematic Liquid Crystals
}

\author{
G. Assanto, M. Peccianti and C. Conti \\ National Institute for the Physics of Matter \\ \&Department of Electronic Engineering \\ Terza University of Rome, Via della Vasca Navale 84, 00146 Rome, Italy \\ We present an overview of our recent experimental results on two- \\ -dimensional optical spatial solitons in voltage biased planar cells with ne- \\ matic liquid crystals. Excitation, induced waveguiding, and interactions are \\ illustrated and interpreted in terms of the inherent re-orientational, non- \\ -resonant and nonlocal nonlinear response.
}

PACS numbers: 42.70.Df, 42.65.Tg

\section{Introduction}

Bright optical spatial solitons, the propagating eigenmodes on a nonlinear system encompassing a self-focusing response counterbalancing diffraction in one or two transverse dimensions, have been long discussed theoretically and numerically, and reported in Kerr-like, quadratic, photorefractive and liquid crystalline media [1-3]. While typical Kerr- and quadratic materials require substantial peak powers and pulsed lasers, however, both photorefractives and liquid crystals exhibit a rather slow but large nonlinear response, and solitons were reported at $\mathrm{mW}$ excitations even with cw-sources [2-4]. This characteristic, in conjunction with their liquid state and inexpensive processing, make liquid crystals quite attractive for soliton investigation in terms of both fundamental phenomena and applications to signal processing. Here we will discuss on excitation, self- and induced-waveguiding, steering and interactions of two-transverse dimension bright spatial solitons in bulk nematic liquid crystals, outlining the role of nonlocality in the nonlinear material response.

\section{Theory}

Nematic liquid crystals contain elongated molecules which, subject to an electric field, undergo a dipole-field torque. The latter tends to alter their initial orientation and reduce the angle between the direction of field oscillation - or 
polarization - and the long molecular axis — or director. As a consequence, field-induced molecular re-orientation results into an increased refractive index according to the usual expression for an extraordinary wave in positive uniaxials, i.e.,

$$
n_{\mathrm{e}}(\theta)=\frac{1}{\sqrt{\frac{\cos ^{2} \theta}{n_{\perp}^{2}}+\frac{\sin ^{4} \theta}{n_{\|}^{2}}}}
$$

with $n_{\|}>n_{\perp}$. Whenever the director-axis is initially orthogonal to the field, however, the field-dependent orientation $\theta$ is subject to a threshold, known as the Freedericks intensity. For an initial $\theta=\theta_{0} \neq 0$ this nonlinear effect becomes Kerr-like with saturation ( $\theta$ can never exceed $\pi / 2$ ), and is indeed maximized for $\theta_{0}=\pi / 4[5,6]$. While a saturating Kerr response ensures existence and stable propagation of bright solitons in two transverse dimensions [7], the elastic forces among the molecules in the medium provide it with nonlocality. The latter, for small enough reorientation values, is the leading stabilizing mechanisms for such solitons [8]. The equations coupling the director reorientation $\psi$ with the optical field $a$ propagating down the crystal can be cast in a dimensionless form as

$$
\mathrm{i} \frac{\partial a}{\partial z}+\nabla_{x y}^{2} a-a+\psi a=0, \quad \epsilon \frac{\partial^{2} \psi}{\partial z^{2}}+\nabla_{x y}^{2} \psi-\alpha \psi+\frac{1}{2}|a|^{2}=0,
$$

where we introduced

$$
\alpha=\frac{\varepsilon_{\mathrm{RF}} E^{2}}{\pi k K \beta}, \quad \epsilon=\frac{\beta}{2 \alpha k},
$$

with $E$ - the amplitude of a (quasi-stationary or low-frequency) electric field providing the pre-tilt $\theta_{0}, \varepsilon_{\mathrm{RF}}$ - the dielectric anisotropy, $A$ - the slowly-varying amplitude of the propagating optical field of wavelength $\lambda=2 \pi n\left(\theta_{0}\right) / k, \beta-$ the nonlinear correction to the wavevector $k, K$ - the Frank elastic constant taken equal for splay, bend and twist of the molecules. $\alpha$ determines the degree of nonlocality of the medium, and $\epsilon$ is a small constant, which can be neglected in our nematic medium. Soliton solutions are obtained from Eqs. (1) by setting $\partial_{z}=0$ and, for $a$ real, their profile is given by

$$
\nabla_{x y}^{2} a-a+\psi a=0, \quad \nabla_{x y}^{2} \psi-\alpha \psi+\frac{1}{2} a^{2}=0 .
$$

which resemble those describing spatial solitons in bulk quadratic crystals, with $a(\psi)$ playing the role of the fundamental (second) harmonic in a frequency doubling interaction [9]. Despite the different underlying physics, such solitary solutions are known to exist and be stable in two transverse dimensions [3].

\section{Experimental and results}

In our experiments, we employed a planar cell with electrodes permitting the pre-tilt through the application of a voltage across $x$, as sketched in Fig. 1. 


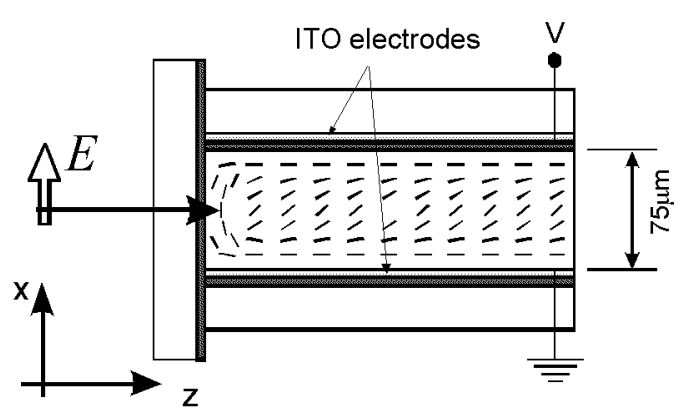

Fig. 1. Planar geometry employed in the experiments. The cell and the spatial distribution of the directors are sketched with the relevant directions. The indium-tin-oxide films allowed the application of a voltage $V$ to pre-tilt the liquid crystal E7.

The glass interfaces were silica-coated to guarantee the planar anchorage of the nematic crystal, and the light beam was injected far from the boundaries and in the $z$-direction.

For an applied voltage of $1 \mathrm{~V}$ at $1 \mathrm{kHz}$, a $2 \mathrm{~mW}$-polarized input beam with a $<2 \mu \mathrm{m}$ waist sufficed to balance diffraction, and a spatial soliton propagating for more than 20 Rayleigh lengths was clearly observed at $514 \mathrm{~nm}$. A comparison between linear and nonlinear propagation regimes is shown in Fig. 2 for the same excitation but using orthogonal linear polarizations, such that the $y$-polarized input, being below the Freedericks threshold, could not induce any nonlinear effects. The photographs were obtained via a microscope, imaging the light scattered above the cell by the beam propagating in the $y-z$ plane. Noteworthy enough, if this polarization dependence clearly identifies the molecular reorientation in the $x-z$ plane as the self-focusing mechanism, it can also be probed by a weaker signal at $633 \mathrm{~nm}$ provided it was co-launched with the beam from the argon laser $(514 \mathrm{~nm})$. Figure 3 shows linear and nonlinear propagation of a probe when co-launched with the soliton-generating beam: it is apparent that the soliton originates a channel waveguide able to confine the weak beam. Such capability is, indeed, wavelength

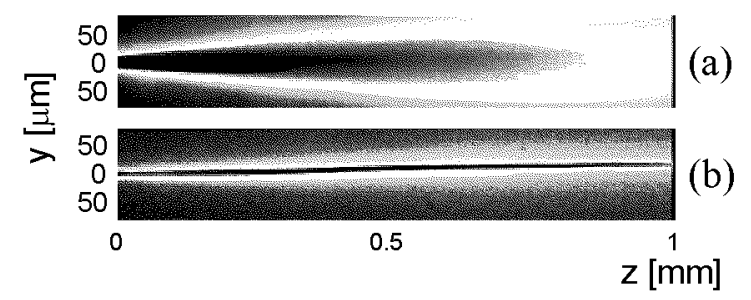

Fig. 2. Photographs of a linearly diffracting (a) and solitary (b) beam of $2 \mathrm{~mW}$ power from an argon-ion laser oscillating at $514 \mathrm{~nm}$. In the upper case the light was polarized orthogonally to the reorientation plane $(x-z)$. 


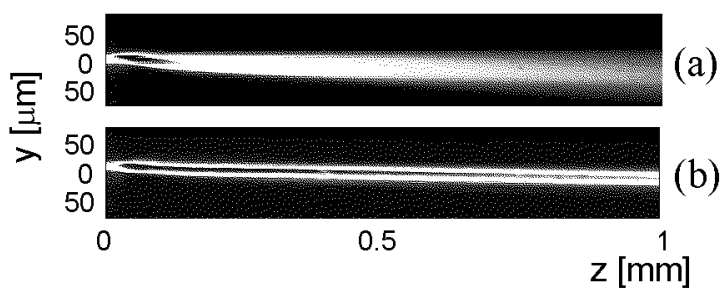

Fig. 3. Photographs of a linearly diffracting (a) and guided (b) beam of $100 \mu \mathrm{W}$ power from a helium-neon laser oscillating at $633 \mathrm{~nm}$, correspondingly to the two cases shown in Fig. 2. The probe was collinearly launched and co-polarized with the argon beam.

and protocol transparent, in-as-much-as the medium response relies on a molecular effect and is non-resonant. Even linear losses, present but largely attributable to scattering, are bound to decrease at longer wavelengths. All-optically induced waveguiding of an otherwise freely diffracting signal allows to envision a number of applications, from signal readdressing in space to logic operations and gating.

Figure 4 shows an example of signal steering by acting on the input angle of the soliton-generating argon beam: an $x$-polarized beam at $514 \mathrm{~nm}$ is injected at two opposite angles with respect to the $z$-direction, while a co-polarized signal at $633 \mathrm{~nm}$ is launched along $z$. The signal is trapped into the soliton and, thanks to the significant numerical aperture of the induced channel, modifies its direction
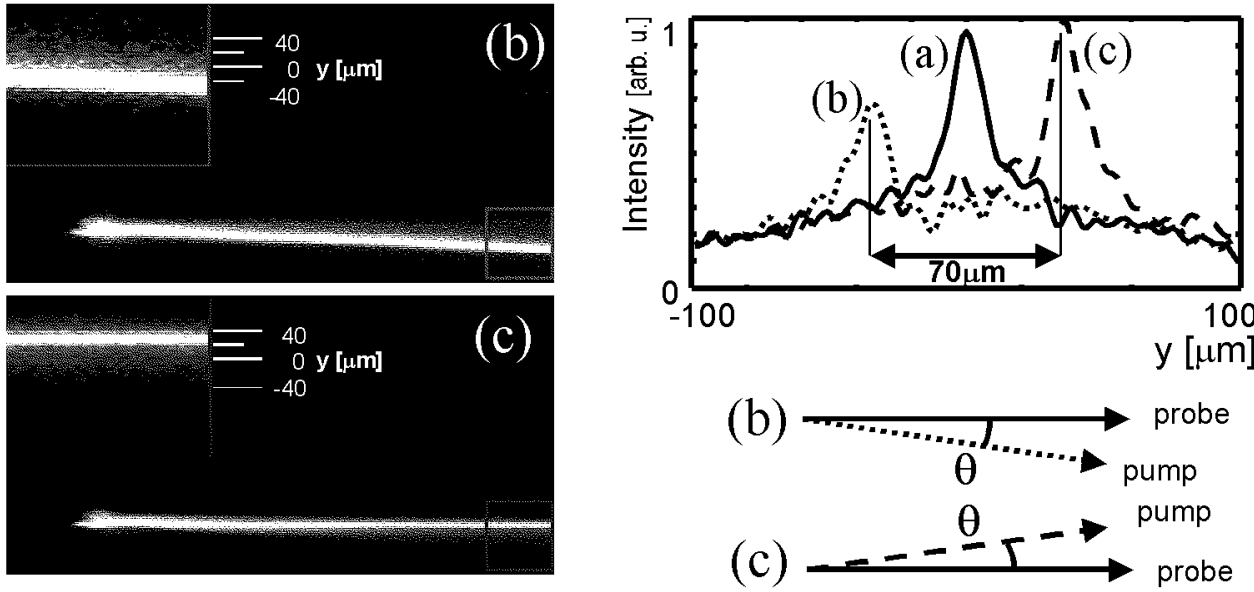

Fig. 4. Experimental results on propagation of a weak $(<20 \mu \mathrm{W}) x$-polarized signal at $633 \mathrm{~nm}$ when tilting by $\theta$ the soliton obtained with a $2.2 \mathrm{~mW} \mathrm{Ar}^{+}$beam: (a) $\theta=0^{\circ}$, (b) $\theta=-2.3^{\circ}$, (c) $\theta=+2.3^{\circ}$. The photographs on the left (and the insets with enlargements) show that some radiated light is present in the last two cases. The graph on the top-right displays the corresponding transverse profiles after $z=1 \mathrm{~mm}$, indicating a lateral shift as large as $70 \mu \mathrm{m}$. 
of propagation as the soliton is steered. After $1 \mathrm{~mm}$, the signal has been laterally shifted in the output plane by as much as $70 \mu \mathrm{m}[10]$.

Noticeably, the feature described above could be effectively implemented by steering the soliton by acting on the applied voltage through a properly tailored electrode or by a phasefront-modulator. Furthermore, Eqs. (2) describe a nonlocal soliton which can also be excited with a spatio-temporal incoherent beam, allowing the all-optical control and the signal addressing to be implemented with light-emitting diodes or white-light sources [11].

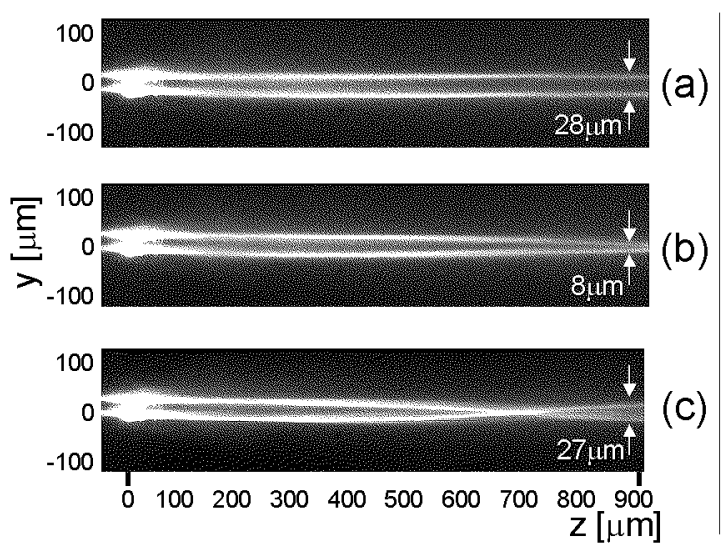

Fig. 5. Interaction of two identical solitons launched with a $28 \mu \mathrm{m}$ separation along $y$ and a $5^{\circ}$ relative angle: (a) weak attraction for a power of $2.8 \mathrm{~mW}$ each; (b) stronger attraction at $3.6 \mathrm{~mW}$; (c) crossing at $4.5 \mathrm{~mW}$. The white arrows on the far right indicate the output separation resulting after the interaction.

The nonlocality, however, is better exploited in soliton-soliton interactions, where it results in a long-range attractive force between them, due to the transverse extension of the refractive perturbation in a liquid governed by elastic forces [12]. Such behavior is clearly illustrated in Fig. 5, where two equi-power beams at $514 \mathrm{~nm}$ are injected at $5^{\circ}$ from one another to form initially-diverging solitons. Due to the mutual attraction, however, their trajectories become nearly parallel at $2.8 \mathrm{~mW}$, bend and converge at $3.6 \mathrm{~mW}$, and cross and interlace at $4.5 \mathrm{~mW}$. Their final separation at $z=0.9 \mathrm{~mm}$, in turn, goes from 28 to 8 and to $-27 \mu \mathrm{m}$, respectively [13]. Naturally, this collisional long-range interaction between spatial solitons excited by the pump is of relevance in terms of a co-launched co-polarized weak probe in one or both of them. A soliton-confined signal, in fact, would be subject to the same trajectory deviations although its power and/or modulation format remains unaffected. An example is shown in Fig. 6, where a power-dependent $\mathrm{X}$-junction can be obtained by launching two solitons parallel to one another: for $1.7 \mathrm{~mW}$ excitation, the solitons proceed straight, and so do the 

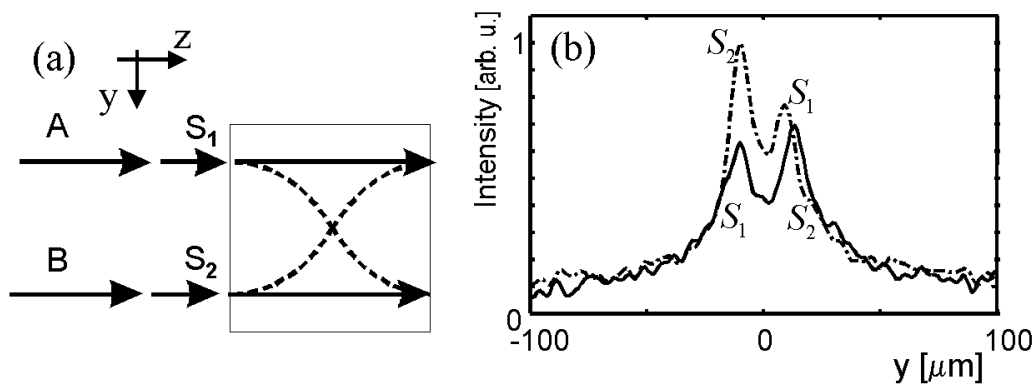

Fig. 6. A power dependent X-junction. (a) Sketch: signals $S_{1}$ and $S_{2}$ at $633 \mathrm{~nm}$ are co-launched with the soliton beams $A$ and $B$, respectively. At low powers (solid trajectories) the signals proceed straight, whereas at high powers (dashes) their output positions are flipped. (b) Experimental signal profiles after $1 \mathrm{~mm}$, at low (solid line) and high (dot-dashes) powers.

signals co-propagating with (one or both of) them. As the input is increased to $4.3 \mathrm{~mW}$, however, the outputs are flipped in space, realizing an X-switch [14].

Finally, interesting possibilities are offered by using three solitons to engineer all-optical logic gates. An example is the NOR gate sketched in Fig. 7a. Two inputs $A$ and $B$ determine the whereabouts of the signal co-launched with and guided by soliton $S$. The latter's trajectory depends on the presence of either one or both $A$ and $B$, which cause its deflection based on long-range attraction. The signal will exit through the output port Out, therefore, only when neither $A$ nor $B$ are injected, effectively realizing a NOR gate, with the truth table shown in the inset on the right. Figure $7 \mathrm{~b}$ displays the actual results of experiments performed with $1.7 \mathrm{~mW}$ input beams, probing the signal transverse profiles after a $1 \mathrm{~mm}$ propagation [14]. The signal peak position is labeled in terms of the logic state of solitons $A$ and $B$ (with " 0 " and "1" corresponding to absence and presence of the soliton, respectively).
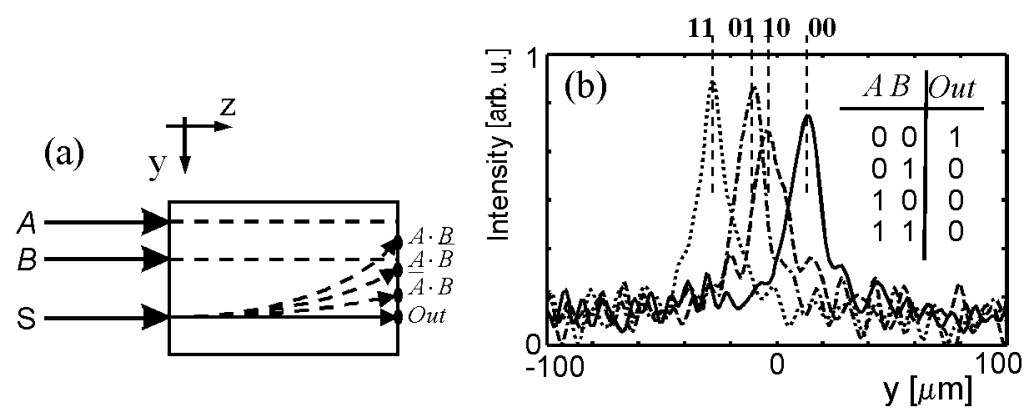

Fig. 7. A NOR gate with three solitons. (a) Sketch: $A$ and $B$ are control inputs at $514 \mathrm{~nm}, S$ guides the signal at $633 \mathrm{~nm}$. (b) Signal transverse profiles obtained after $1 \mathrm{~mm}$ for various input combinations. The truth table in the inset refers to the Out port. 


\section{Conclusion}

In conclusion, solitons in nematic liquid crystals are an excellent test-bed for all-optical processing and switching based on light self- and cross-localization. Although the reported effects are inherently slow, faster response times can be pursued in different materials and/or geometries, leading the way to applications for optical communications.

\section{Acknowledgments}

The authors thank Prof. G.I. Stegeman and Prof. I.C. Khoo for enlightening discussions, Prof. C. Umeton and A. De Luca for the samples, K.A. Brzdąkiewicz for some of the numerical simulations, and the Italian Institute for the Physics of Matter for financial support through PRA 2002 "SPASONELIC" and M.P.'s scholarship.

\section{References}

[1] G.I. Stegeman, D.N. Christodoulides, M. Segev, IEEE J. Sel. Top. Quantum Electron. 6, 1419 (2000).

[2] Soliton Driven Photonics, Ed. A.D. Boardman, Kluwer Academic Publ., Dordrecht 2001.

[3] Spatial Solitons, Eds. S. Trillo, W. Torruellas, Springer, Berlin 2001.

[4] M. Peccianti, G. Assanto, A. de Luca, C. Umeton, I.C. Khoo, Appl. Phys. Lett. $77,7(2000)$.

[5] N.V. Tabiryan, A.V. Sukhov, B.Ya. Zel'dovich, Mol. Cryst. Liq. Cryst. 136, 1 (1986).

[6] I.C. Khoo, Liquid Crystals: Physical Properties and Nonlinear Optical Phenomena, Wiley, New York 1995.

[7] P.L. Kelley, Phys. Rev. Lett. 15, 1005 (1965); D. Grishkowsky, Phys. Rev. Lett. 24, 866 (1970); J.E. Bjorkholm, A. Ashkin, Phys. Rev. Lett. 32, 129 (1974).

[8] S.K. Turitsyn, Theor. Math. Phys. 64, 797 (1985).

[9] C. Conti, M. Peccianti, G. Assanto, in: OSA Trends in Optics and Photonics (TOPS), Nonlinear Guided Waves and Their Applications, Vol. 80, OSA Technical Digest, Optical Society of America, Washington DC 2001, p. NLMD32-1.

[10] M. Peccianti, G. Assanto, Opt. Lett. 26, 1690 (2001).

[11] M. Peccianti, G. Assanto, Opt. Lett. 26, 1791 (2001).

[12] A.W. Snyder, D.J. Mitchell, Science 276, 1538 (1997); D. Mitchell, A.W. Snyder, J. Opt. Soc. Am. B 16, 236 (1999).

[13] M. Peccianti, K.A. Brzdąkiewicz, G. Assanto, Opt. Lett. 27, 1460 (2002).

[14] M. Peccianti, C. Conti, G. Assanto, A. De Luca, C. Umeton, Appl. Phys. Lett. $81,3335(2002)$. 